\title{
Workshop on Information Retrieval over Social Networks
}

\author{
Stephane Marchand-Maillet ${ }^{1}$, Arjen P. de Vries ${ }^{2}$, and Mor Naaman ${ }^{3}$ \\ 1 University of Geneva, Switzerland \\ 2 CWI/TU Delft, The Netherlands \\ 3 Rutgers University, USA
}

Popular online communities and services such as Flickr, Youtube, Facebook or LinkedIn are spearheading an emerging type of information on the Web. This information is composed of classical textual and multimedia data, in concert with additional data (tags, annotations, comments, ratings). Perhaps most significantly, the information is overlaid on an explicit social network created by the participants of each of these communities. The result is a rich structure of interrelationships between content items, participants and services. Although the size of such networks requires the use of advanced Information Retrieval techniques, classical IR models are not tailored for this type of content as they do not (in general) take advantage of the particular structure and unique aspects of this socially-driven content. This workshop proposes to report about the state-ofthe-art in this direction and to gather a relevant panel of researchers working in the field. This workshop will consist of research papers that address Information Retrieval over Social Networks, including:

- Applications of Information Retrieval over Social Network

- Adapted IR models for Social Networks

- Mining Social Network data

- Privacy issues in Social Network information retrieval

- Trust and Reliability issues in Social Network information retrieval

- Knowledge and Content Discovery in Social Networks

- Information diffusion over Social Networks

- Performance evaluation for the above (measures, test collections) 